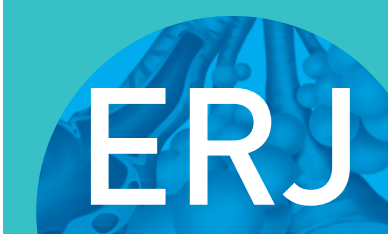

open research

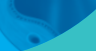

\title{
A retrospective analysis of outcomes in low- and intermediate-high-risk pulmonary embolism patients managed on an ambulatory medical unit in the UK
}

\author{
Michael E. Reschen ${ }^{1}$, Jonathan Raby ${ }^{1}$, Jordan Bowen ${ }^{1}$, Sudhir Singh ${ }^{1}$, \\ Daniel Lasserson ${ }^{2}$ and Christopher A. O'Callaghan ${ }^{3}$
}

Affiliations: 1 Dept of Acute General Medicine, John Radcliffe Hospital, Oxford University NHS Hospitals Foundation Trust, Oxford, UK. ${ }^{2}$ Institute of Applied Health Research, College of Medical and Dental Sciences, University of Birmingham, Birmingham, UK. ${ }^{3}$ Wellcome Trust Centre for Human Genetics, University of Oxford, Oxford, UK.

Correspondence: Michael E. Reschen, Dept of Acute General Medicine, John Radcliffe Hospital, Oxford University NHS Hospitals Foundation Trust, Headley Way, Oxford, OX3 9DU, UK. E-mail: michael.reschendouh.nhs.uk

ABSTRACT Pulmonary embolism (PE) is common and guidelines recommend outpatient care only for PE patients with low predicted mortality. Outcomes for patients with intermediate-to-high predicted mortality managed as outpatients are unknown.

Electronic records were analysed for adults with PE managed on our ambulatory care unit over 2 years. Patients were stratified into low or intermediate-to-high mortality risk groups using the Pulmonary Embolism Severity Index (PESI). Primary outcomes were the proportion of patients ambulated, 30-day allcause mortality, 30-day PE-specific mortality and 30-day re-admission rate.

Of 199 PE patients, $74 \%$ were ambulated and at 30 days, all-cause mortality was $2 \%$ (four out of 199) and PE-specific mortality was $1 \%$ (two out of 199). Ambulated patients had lower PESI scores, better vital signs and lower troponin levels (morning attendance favoured ambulation). Over a third of ambulated patients had an intermediate-to-high risk PESI score but their all-cause mortality rate was low at $1.9 \%$ (one out of 52). In patients with intermediate-to-high risk, oxygen saturation was higher and pulse rate lower in those who were ambulated. Re-admission rate did not differ between ambulated and admitted patients.

Two-thirds of patients with intermediate-to-high risk PE were ambulated and their mortality rate remained low. It is possible for selected patients with intermediate-to-high risk PESI scores to be safely ambulated.

@ERSpublications

In a cohort of 199 patients with pulmonary embolism (PE), 52 were managed as outpatients despite an intermediate-to-high risk of mortality. Only 1 outpatient died within 30 days and not from PE, suggesting opportunities for increased ambulatory care. http://ow.ly/4p4D30oaNkr

Cite this article as: Reschen ME, Raby J, Bowen J, et al. A retrospective analysis of outcomes in low- and intermediate-high-risk pulmonary embolism patients managed on an ambulatory medical unit in the UK. ERJ Open Res 2019; 5: 00184-2018 [https://doi.org/10.1183/23120541.00184-2018].

Received: Oct 152018 | Accepted after revision: Feb 282019

Copyright $\odot E R S$ 2019. This article is open access and distributed under the terms of the Creative Commons Attribution Non-Commercial Licence 4.0. 


\section{Introduction}

Pulmonary embolism (PE) is potentially fatal and accounted for over 27000 hospital admissions in the UK in 2011 and consumption of around 250000 bed days [1]. The success of anticoagulant treatments that can be safely administered intermittently out of hospital has made outpatient management of PE a technical possibility [2]. Managing PE on an outpatient basis could reduce bed-occupancy, reduce healthcare costs and improve patient satisfaction, without affecting safety or efficacy [3].

Acute PE is heterogenous in its severity and scoring systems have been developed and validated to stratify risk of mortality, recurrence and major bleeding [4-6]. The Pulmonary Embolism Severity Index (PESI) score is calculated from 11 clinical parameters to determine the risk of mortality at 30 days and has been extensively validated [4, 7-9]. The PESI scoring system classifies patients into five levels, with levels one and two grouped as "low risk" (score <86, 30-day mortality 0-1\%) and levels three, four and five grouped as "intermediate-to-high risk" (score $\geqslant 86,30$-day mortality 3.1-23.9\%) [4, 10]. A randomised controlled trial demonstrated non-inferiority of outpatient management for patients with a low risk PESI score [7]. The European Society of Cardiology (ESC) and the British Thoracic Society (BTS) used this evidence in their recent guidelines on PE management to recommend that patients with a low risk PESI score be considered for outpatient management and patients with intermediate-to-high risk be admitted [11, 12]. For example, the ESC guidelines recommend that patients with intermediate-to-high risk PESI scores be admitted for close monitoring in case rescue reperfusion therapy is required; however, no mortality benefit from this approach has been demonstrated $[11,13]$. Nevertheless, there is an assumption that a predicted mortality threshold exists, whereby inpatient care may be safer and there is a lack of evidence as to whether some patients with intermediate or high risk PESI scores may be safely managed on an outpatient basis.

The introduction of ambulatory care units in the UK has provided an opportunity for the management of PE on an outpatient basis, allowing for diagnosis, monitoring and initiation of treatment over a period of several hours. At our ambulatory medical unit within a major teaching hospital we operated an inclusive acceptance policy for referrals with suspected PE, whereby a senior ambulatory clinician triaged referrals without rigid exclusion criteria. Our institutional guidelines allowed for patients with non-massive PE and intermediate-to-high risk PESI scores to be managed on an ambulatory basis if this was determined to be appropriate after shared decision-making between the medical consultant and the patient. In this study we report the clinical characteristics and outcomes for all patients diagnosed with an acute PE who were managed on the ambulatory medical unit. We present the outcomes in this cohort, stratified by PESI score.

\section{Methods}

We operated an inclusive referral system to the ambulatory assessment unit (AAU), accepting all adult patients for whom ambulation was considered likely. Referrals were triaged by the AAU consultant physician or the medical registrar during the day and by the senior house officer overnight. Direct referrals were accepted from the emergency department, general practitioners, paramedics, specialist nurses, community hospital based ambulatory units and outpatient clinics. Additionally, non-ambulatory patients residing in community hospitals could also be referred for urgent cross-sectional imaging on a day-case basis when there was no local imaging availability. Patients with suspected acute PE or incidental findings of PE were investigated and managed according to the hospital's venous thromboembolism protocol.

We ascertained all patient episodes on the AAU with a primary diagnosis code of "pulmonary embolism without cor pulmonale" between January 01, 2016 and December 21, 2017 (there were no episodes where the presence of PE-related cor pulmonale was coded). For patients who had more than one episode with a primary diagnosis of PE we included only the first episode, giving a total of 275 patients. We excluded patients who were either miscoded with the primary diagnosis of PE or who were transiently accommodated on the AAU during their discharge from an inpatient unit bed, resulting in the exclusion of 76 subjects. To evaluate the trajectory and safety of care for patients with PE, we assessed the primary outcomes: ambulation status at discharge, all-cause mortality at 30 days, PE-specific mortality at 30 days and re-admission rate at 30 days (including ambulatory unit attendances but excluding emergency department attendances not leading to admission).

We also ascertained baseline demographic parameters, co-morbidities, markers of PE severity using the PESI scoring system and the extent of PE on imaging and with biomarkers (troponin and brain natriuretic protein (BNP)) [4]. For clinical observations, the first recorded value on the AAU was used. The decision to ambulate patients who had been accepted onto the AAU and diagnosed with PE was made by the AAU consultant physician. We collected data from the hospital electronic patient record, collated the data in Microsoft Excel (Microsoft Corp, Redmond, WA, USA) and analysed the data using the Pandas module (https://pandas.pydata.org). Statistical analysis was performed using Fisher's exact test for categorical data, where one or more cells of the contingency table contained less than five outcomes, and the Chi-squared test with Yates correction for all other cases. For continuous variables an unpaired two-tailed t-test was 
performed. The p-value threshold for significance was set at 0.05 . The study was approved by the hospital clinical governance department and was not deemed to need additional research ethics approval because it was a retrospective service evaluation.

\section{Results}

Clinical characteristics and mortality in AAU patients diagnosed with PE

We identified 199 patients with a primary diagnosis of PE who were managed on the AAU. Of these, 95 $(47.7 \%)$ were male and the mean age was 62.3 years with a range from 19-93 years. The D-dimer was abnormal in all 147 tested patients. Computed tomography pulmonary angiography (CTPA) was performed in 189 patients (95.0\%) and nuclear perfusion scintigraphy was performed in a further nine patients (4.5\%). One patient had a fatal cardiac arrest before imaging could be completed. PE was an incidental finding on computed tomography (CT) scanning performed for other indications in 13 patients (6.5\%). The number of ambulated patients was $148(74 \%)$ and 30-day all-cause mortality was $2 \%$ (four out of 199 patients), while 30-day PE-specific mortality was $1 \%$ (two out of 199 patients). The 30-day re-admission rate was $9 \%$ (18 out of 199 patients). Data for mortality risk stratification using the PESI tool were available for 187 patients (94\%), which included all patients who died by 30 days. The mean, minimum and maximum PESI scores were 82.8, 19 and 199, respectively. When patients were stratified by PESI score, 30-day all-cause mortality was $0.94 \%$ (one out of 106) in low risk patients and $3.7 \%$ (three out of 81 ) in intermediate-to-high risk patients. The corresponding PE-specific mortality rates were $0 \%$ and $2.5 \%$ (two out of 81 patients).

\section{Clinical characteristics and severity status of ambulated versus admitted PE patients}

Admitted patients had significantly higher pulse rate, greater use of oxygen, lower oxygen saturation and lower temperature than ambulated patients (table 1). The PESI score was significantly higher in admitted patients; however, there was no difference in the proportion of patients (ambulated or admitted) who had an intermediate-to-high risk PESI score. Using the current BTS guideline criteria for admission (based on PESI score) there would have been 52 more admissions to hospital, a doubling of the admission rate [12]. There was no significant difference in re-admission rate or mortality in admitted patients; however, the troponin level was significantly higher in admitted patients. We examined whether a greater proportion of patients arriving on the AAU in the morning (before 13:00) were ambulated compared to those who arrived after 13:00. We found that a significantly higher proportion of the patients seen in the morning were ambulated.

\section{Radiological findings in ambulated versus admitted patients}

There were no significant differences in radiological findings between admitted and ambulated patients (table 2). There was also no difference in the proportion of patients who had been diagnosed with PE incidentally, having had a CT scan for an alternative reason such as cancer staging follow-up.

\section{TABLE 1 Characteristics of ambulated and admitted patients}

\begin{tabular}{|c|c|c|c|}
\hline Variable & Ambulated $(n=136)$ & Admitted ( $n=51)$ & p-value \\
\hline Age years (mean) & 61.3 & 65.8 & 0.14 \\
\hline Male gender & $65(47.8)$ & $23(45.1)$ & 0.87 \\
\hline Pulse rate beats $\cdot \min ^{-1}$ & 82.4 & 93.1 & 0.0001 \\
\hline Systolic BP mmHg & 137.3 & 136.2 & 0.73 \\
\hline Oxygen saturation $\%$ & 95.9 & 93.6 & 0.000005 \\
\hline Use of oxygen & $2(1.5)$ & 19 (37.3) & 0.0001 \\
\hline Respiratory rate breaths $\cdot \min ^{-1}$ & 17.8 & 20.0 & 0.0001 \\
\hline Temperature ${ }^{\circ} \mathrm{C}$ & 36.4 & 36.6 & 0.03 \\
\hline Altered mental status & $0(0)$ & $1(1.8)$ & 0.27 \\
\hline Heart failure & $6(4.4)$ & 7 (13.7) & 0.057 \\
\hline Cancer & 27 (19.9) & $9(17.6)$ & 0.89 \\
\hline Lung disease & $26(19.1)$ & $13(25.5)$ & 0.45 \\
\hline PESI score & 79.6 & 92.0 & 0.014 \\
\hline Intermediate-to-high risk patients & $52(38.2)$ & $29(56.9)$ & 0.66 \\
\hline Troponin $\mathrm{ng} \cdot \mathrm{mL}^{-1}$ & $0.003(n=79)$ & $0.087(n=34)$ & $0.7 \times 10^{-6}$ \\
\hline Morning arrival & $91(66.9)$ & 19 (37.3) & 0.0005 \\
\hline Re-admission rate & $13(9.6)$ & $2(3.9)$ & 0.36 \\
\hline All-cause mortality & $1(0.7)$ & $3(5.9)$ & 0.0625 \\
\hline
\end{tabular}

Data is presented as $n$ or $n(\%)$ unless otherwise stated. BP: blood pressure. PESI: Pulmonary Embolism Severity Index. 
Further analysis of the subset of PESI score determined intermediate-to-high risk patients We ambulated 52 patients with PESI scores in the intermediate-to-high risk category. A further 29 patients with intermediate-to-high risk PE were admitted. The mean PESI scores in the ambulated and admitted patients were 110.8 and 114.1, respectively, both being in the category of high risk (PESI score 106-125) (table 3). Admitted patients had significantly higher pulse rates and respiratory rates, with lower oxygen saturations, and were more likely to be using supplemental oxygen. This suggests physicians place a higher value on physiological parameters of risk than cancer in deciding on when to admit patients.

\section{Mortality in ambulated and admitted patients}

To further assess the factors associated with mortality at 30 days in our cohort, we checked the causes of death and the timing of mortality in relation to assessment on the AAU (table 4). Only one of the four patients who died had been ambulated and the other three patients were admitted. The ambulated patient had metastatic cancer and died of sepsis 16 days after assessment on the AAU. Of the three patients who were admitted from the AAU, two died from PE the day following admission and one died from pulmonary fibrosis 21 days later. These data suggest death from PE is uncommon and, notably, both deaths from PE occurred within $48 \mathrm{~h}$ of presentation at the AAU.

Finally, we compared the clinical and radiological features of the patients who had died within 30 days to those of survivors, irrespective of ambulation status (table 5). Overall, there were no statistically significant differences although the small number of deaths limited the power of this analysis. However, there were trends towards deceased patients being older, having a higher PESI score, greater oxygen use and pre-existing lung disease.

\section{Discussion}

The development of ambulatory medicine units in the UK has expanded, with a recent survey of 132 acute care hospitals finding that over $90 \%$ have an ambulatory emergency care service [14]. Whereas lower limb deep vein thrombosis (DVT) is almost always managed as an outpatient, traditional practice has been to admit patients with PE, mainly due to the risk of deterioration and death [15]. The typical duration of admission for PE is about 3 to 6 days $[16,17]$. The introduction of directly acting oral anticoagulants has facilitated the outpatient management of PE by removing the need for cumbersome inpatient titration of warfarin [18]. The safe utilisation of ambulatory care services for patients with suspected PE could, amongst other benefits, reduce hospital bed usage. Guidelines from the BTS published in 2018 recommend ambulation for patients with low predicted mortality risk as defined by a validated clinical scoring tool, but admission for patients with intermediate-to-high predicted mortality risk [12]. We examined the outcomes of nearly 200 patients with PE who were assessed on our AAU, with particular attention to the outcomes of intermediate-to-high risk patients who were ambulated.

We ambulated about $75 \%$ of PE patients, in keeping with the expected $60-90 \%$ range documented in the Directory of Ambulatory Emergency Care produced by the National Health Service (NHS) Ambulatory Emergency Care Network [19]. From our cohort of ambulated patients more than a third were classed as intermediate-to-high risk based on the PESI scoring system (the most validated and widely-used risk stratification tool) [4].

The approach advised in the recent BTS guideline is that patients with an intermediate-to-high risk PESI score are admitted to hospital [12]. The basis for admitting these patients is that a higher PESI score indicates a greater mortality risk and there is an assumption that admission might in some way mitigate this risk. This accords with the ESC guidelines that recommend admission for patients with intermediate-to-high risk PE. However, this recommendation is based on a single randomised study of

TABLE 2 Radiological findings in ambulated and admitted patients

\begin{tabular}{lccc} 
Radiological finding & Ambulated & Admitted & p-value \\
\hline Bilateral $^{\#}$ & $91 / 134(67.9)$ & $38 / 49(77.6)$ & 0.28 \\
RV strain" & $24 / 129(18.6)$ & $15 / 50(30)$ & 0.15 \\
Saddle embolus $^{\text {"1 }}$ & $3 / 129(2.3)$ & $1 / 50(2)$ & 1.0 \\
Sub-segmental" $^{\text {In }}$ & $12 / 128(9.4)$ & $3 / 50(6)$ & 0.67 \\
Incidental finding" $^{\text {Th }}$ & $8 / 136(5.9)$ & $2 / 50(4)$ & 1.0
\end{tabular}

Data is presented as $\mathrm{n} / \mathrm{n}(\%) . \mathrm{RV}$ : right-ventricular. ${ }^{\#}$ : finding based on computed tomography pulmonary angiography (CTPA) or nuclear perfusion scanning; ${ }^{\text {": }}$ finding based on CTPA. 
TABLE 3 Comparison of patients with a non-low risk Pulmonary Embolism Severity Index (PESI) score who were ambulated or admitted

\begin{tabular}{|c|c|c|c|}
\hline Variable & Ambulated $(n=52)$ & Admitted (n=29) & p-value \\
\hline Age years (mean) & 74.6 & 76.2 & 0.53 \\
\hline Male gender & $28(53.8)$ & $11(38)$ & 0.63 \\
\hline Pulse rate beats $\cdot \mathrm{min}^{-1}$ & 84.3 & 95 & 0.003 \\
\hline Systolic BP mmHg & 136.9 & 137.4 & 0.91 \\
\hline Oxygen saturation $\%$ & 95.3 & 92.4 & 0.001 \\
\hline Use of oxygen & $2(3.8)$ & 12 (41.3) & 0.0007 \\
\hline Respiratory rate breaths $\cdot \min ^{-1}$ & 18 & 20.9 & 0.004 \\
\hline Temperature ${ }^{\circ} \mathrm{C}$ & 36.2 & 36.5 & 0.069 \\
\hline Altered mental status & $0(0)$ & $1(3.4)$ & 0.36 \\
\hline Heart failure & $5(9.6)$ & $7(24.1)$ & 0.15 \\
\hline Cancer & $26(50)$ & 9 (31) & 0.11 \\
\hline Lung disease & $20(38.4)$ & 11 (38) & 0.85 \\
\hline PESI score & 110.8 & 114.1 & 0.51 \\
\hline Troponin $\mathrm{ng} \cdot \mathrm{mL}^{-1}(\mathrm{n}=22)$ & 0.01 & 0.07 & 0.06 \\
\hline
\end{tabular}

non-shocked PE patients, with either right-ventricular (RV) dysfunction or a positive troponin test, in which thrombolysis did not improve mortality compared to standard care $[11,13]$. The evidence-based rationale for admission is therefore unclear and, in the absence of compelling evidence for specific admission criteria, the BTS guideline threshold for admission would appear to be based on an arbitrary mortality threshold-this parallels the use of the popular CURB-65 mortality risk score, which is used as a criterion for admission in community-acquired pneumonia (CAP) [20]. Patients with a CURB-65 score of $0-1$, which confers a mortality rate of less than $3 \%$, are considered potentially suitable for ambulation [21]. In our study, one of the 52 patients ambulated with intermediate-to-high risk died within 30 days. This mortality rate is in line with that of the low risk cohort from the study in which the PESI tool was derived [4]. Furthermore, in our study, the cause of death was unrelated to PE and this may indicate that within the intermediate-to-high risk group physicians are able to identify patients who are at a low risk of death. We found that within the intermediate-to-high risk cohort the ambulated patients had significantly better heart rates, respiratory rates and oxygen saturations, and less need for oxygen, despite having a similar PESI score. This suggests that clinicians place emphasis on basic vital signs in making decisions on ambulation.

Other factors that could be used to further stratify risk in patients with intermediate-to-high risk PESI scores include radiological markers of cardiac compromise or biochemical measurements of troponin and BNP. The presence of right-heart strain on CTPA had no effect on the decision to ambulate and did not influence mortality. This is consistent with the finding that, in normotensive PE patients (representing 95\% of PE patients, PESI score not reported), RV dilatation (a measure of RV dysfunction) was not associated with increased mortality [22]. In another study, which did not stratify patients using a risk

TABLE 4 Detailed analysis of patients who died within 30 days of ambulatory assessment unit attendance

\begin{tabular}{|c|c|c|c|c|c|c|c|}
\hline Patient & $\begin{array}{l}\text { Admission } \\
\text { status }\end{array}$ & $\begin{array}{c}\text { Age } \\
\text { years } \\
\text { (mean) }\end{array}$ & $\begin{array}{l}\text { PESI } \\
\text { score }\end{array}$ & Comorbidity & $\begin{array}{l}\text { Cause of } \\
\text { death }\end{array}$ & $\begin{array}{c}\text { Time to } \\
\text { death } \\
\text { days }\end{array}$ & Scan \\
\hline 1 & Admitted & 70 & 70 & Nil & PE & 1 & No scan \\
\hline 2 & Admitted & 80 & 103 & Lung & $\begin{array}{l}\text { Pulmonary } \\
\text { fibrosis }\end{array}$ & 21 & $\begin{array}{c}\text { Bilateral } \\
\text { sub-segmental }\end{array}$ \\
\hline 3 & Admitted & 83 & 130 & Lung & PE & 1 & Bilateral \\
\hline 4 & Ambulated & 58 & 118 & $\begin{array}{c}\text { Metastatic } \\
\text { cancer }\end{array}$ & Sepsis & 16 & Unilateral \\
\hline
\end{tabular}

PESI: Pulmonary Embolism Severity Index; PE: pulmonary embolism. 
TABLE 5 Comparison of severity factors in surviving patients and patients deceased within 30 days of ambulatory assessment unit attendance with pulmonary embolism (PE)

\begin{tabular}{|c|c|c|c|}
\hline Variable & Surviving ( $n=183$ ) & Deceased ( $n=4)$ & p-value \\
\hline Age years (mean) & 62.3 & 72.75 & 0.25 \\
\hline PESI score & 82.3 & 105.2 & 0.15 \\
\hline Cancer & $35(19.1)$ & $1(25)$ & 0.58 \\
\hline Heart failure & $13(7.1)$ & $0(0)$ & 1.0 \\
\hline Lung disease & $37(20)$ & $2(50)$ & 0.19 \\
\hline Ambulated & $135(74)$ & $1(25)$ & 0.063 \\
\hline Troponin $\mathrm{ng} \cdot \mathrm{mL}^{-1}$ & $0.027(n=110)$ & $0.07(n=3)$ & 0.40 \\
\hline $\mathrm{D}$-dimer $\mathrm{ng} \cdot \mathrm{mL}^{-1}$ & 6120 & 9261 & 0.54 \\
\hline Oxygen use & $19(10.4)$ & $2(50)$ & 0.063 \\
\hline Saddle embolus & 4/176 (2.3) & $0 / 3(0)$ & 1.0 \\
\hline Right-heart strain & $39 / 175$ (22.3) & $0 / 3(0)$ & 1.0 \\
\hline Bilateral PE & $127 / 180(70.1)$ & $2 / 3(66.7)$ & 1.0 \\
\hline Sub-segmental & 14/175 (8) & $1 / 3$ (33.3) & 0.23 \\
\hline Incidental finding & $10 / 183$ (5.5) & $0 / 4(0)$ & 1.0 \\
\hline
\end{tabular}

calculator, there was no effect of clot burden on short-term mortality; however, RV dysfunction was associated with mortality [23].

In many cases a troponin test may be sent as part of the initial diagnostic work-up of chest pain to help exclude a myocardial infarction. Troponin level was measured in most patients in our cohort and we found that, using a standard troponin assay (not high sensitivity), the mean troponin level was only slightly above the threshold for positivity (in keeping with the BTS guideline recommendation that a substantially raised troponin should prompt consideration of an alternative diagnosis other than PE) [12]. A meta-analysis of nine studies showed that the standard troponin assay is not a useful discriminator of mortality risk in normotensive PE patients [24]. Nevertheless, the troponin level was significantly lower in our ambulated patients, which is consistent with clinicians regarding troponin as a useful biomarker in ambulatory decision making.

The use of BNP was infrequent in our cohort of patients, likely in part because the turnaround time was several days for a laboratory measurement and near patient testing was only latterly introduced. A meta-analysis of nine studies investigating the association of BNP and mortality in PE patients without cardiogenic shock found that a normal BNP level had a 99\% negative predictive value (NPV) for short term death [25]. Future studies evaluating the use of BNP levels in making ambulation decisions for patients with intermediate-to-high risk PESI scores may be valuable.

In addition to clinical features, we also found that the time of day when the patient was assessed affected the admission rate. Patients were more likely to have been ambulated if they arrived before 13:00. This may reflect a greater period of time to observe patients arriving early in the day and so greater clinician confidence that the patient trajectory was trending to improvement. A range of other factors may affect the ambulation rate during the day. Staff may be fresher and more able to make challenging decisions in the morning and morning assessment may give time for other social aspects of care to be arranged. Some patients may have been seen and given an immediate treatment dose of anticoagulant the previous day in the emergency department or at the out of hours GP practise, and then brought back for an assessment on the ambulatory unit the next morning. In effect these patients would have already demonstrated a period of stability that would have been reassuring. Further studies are needed to assess if this is a general trend across other diseases seen on ambulatory care units.

For patients who have been admitted from the ambulatory unit, another clinical challenge is deciding when patients can be safely discharged once they no longer require treatment that requires hospital admission (such as supplemental oxygen). In this regard, a notable finding in our study was that deaths from PE occurred within 1-2 days of admission. We studied mortality at 30 days as it is highly unlikely that a potentially ambulatory patient would be admitted for PE for longer than 30 days under most circumstances. As such this timeframe covers the period of key relevance to the acute management problem facing clinicians when selecting patients for ambulation. The PESI score is a prediction tool for 30-day mortality and most PE studies report only 30-day mortality and do not provide more granular data 
on time to mortality. In a study assessing daily mortality out to 5 days in 298 patients hospitalised with PE there was one death and this occurred on day four [16]. It would be helpful if daily PE death rates are reported in future studies.

Beyond the risk of mortality, a further concern about ambulating more patients with PE is whether this adversely affects the re-admission rate. Reassuringly, our analysis found that this was not the case for re-admissions up to 30 days following diagnosis. This is consistent with a randomised controlled trial of ambulatory management for low risk PE, which found no difference in unplanned healthcare utilisation by 90 days [7].

\section{Limitations}

Our study has a number of limitations that could be usefully addressed in the future. We analysed the first observations recorded when the patient arrived on the AAU and were therefore not able to assess rapid trends in improvement or deterioration that would have affected the decision to ambulate. On our unit, ambulatory care visits can span several hours and this could have provided the opportunity to observe trends in clinical parameters that facilitated the selection of suitable patients for ambulation. Although little is known about the impact of changes in the PESI score within a several hour period, by $48 \mathrm{~h}$ the PESI score is reduced to the low-risk category in $27.3 \%$ of patients who are intermediate-to-high risk at diagnosis [26]. Patients with a falling PESI score have a substantially lower mortality than those whose score remains elevated [26]. In some patients, PE can be associated with severe pleuritic chest pain that requires strong opioid analgesia. This is a criterion for which admission is recommended [12]; however, we did not assess pain or analgesia usage in our study. The retrospective design of our study means that it is not possible to ascertain all the factors that were used in the referral for consideration of ambulatory care, or in the decision to ambulate a patient. Several other factors that we did not assess may affect ambulation status including social support status and concurrent active illnesses. We applied the PESI score retrospectively and therefore we cannot determine precisely how the score was or was not used at the time of the patient being assessed. Prospective studies would be useful to analyse the basis of this decision making in real time. Echocardiography was not routinely used in the initial work-up of patients with PE. It is possible that some patients had echocardiography, but this data was not collected. In general, patients were referred to our ambulatory unit because the referring clinician and/or the receiving ambulatory physician believed that there was a reasonable prospect of ambulation and we did not have formal referral criteria. For this reason, our results may not be generalisable to all cases of PE, for example, those in critically ill patients.

We have received positive feedback from patients about their ambulatory care, but we have not systematically studied the impact of our approach on patient satisfaction. The number of times a patient is asked to return to the hospital for follow-up appointments might affect satisfaction, but we did not assess this in this study. Published reports indicate that patient satisfaction is generally high among PE patients treated as outpatients $[7,27,28]$.

\section{Conclusions}

We operated an inclusive approach to acceptance of referrals to the AAU for suspected PE and this led to over a third of patients presenting with clinical features for which hospital admission is recommended under current guidelines. However, $64 \%$ of these patients were ambulated rather than admitted and the mortality rate remained low. The data demonstrate that selected patients with intermediate-to-high risk PESI scores can be safely ambulated if the patient is involved in shared decision-making regarding the level of acceptable risk. Our study supports the development of future prospective studies designed to aid clinicians in selecting patients who have intermediate-to-high risk PESI scores, but who may still be safely ambulated, thereby increasing the opportunity for outpatient treatment of PE.

Conflict of interest: None declared.

\section{References}

1 Noel Snell DS, Hubbard R, Gibson J, et al. Burden of lung disease in the UK; findings from the British Lung Foundation's "respiratory health of the nation" project. Eur Respir J 2016; 48: PA4913.

2 Eldredge JB, Spyropoulos AC. Direct oral anticoagulants in the treatment of pulmonary embolism. Curr Med Res Opin 2018; 34: 131-140.

3 Walen S, Katerberg B, Boomsma MF, et al. Safety, feasibility and patient reported outcome measures of outpatient treatment of pulmonary embolism. Thromb Res 2017; 156: 172-176.

4 Aujesky D, Obrosky DS, Stone RA, et al. Derivation and validation of a prognostic model for pulmonary embolism. Am J Respir Crit Care Med 2005; 172: 1041-1046.

5 Weeda ER, Kohn CG, Fermann GJ, et al. External validation of prognostic rules for early post-pulmonary embolism mortality: assessment of a claims-based and three clinical-based approaches. Thromb J 2016; 14: 7. 
6 Chan CM, Woods C, Shorr AF. The validation and reproducibility of the pulmonary embolism severity index. J Thromb Haemost 2010; 8: 1509-1514.

7 Aujesky D, Roy PM, Verschuren F, et al. Outpatient versus inpatient treatment for patients with acute pulmonary embolism: an international, open-label, randomised, non-inferiority trial. Lancet 2011; 378: 41-48.

8 Fraga M, Taffé P, Méan M, et al. The inter-rater reliability of the Pulmonary Embolism Severity Index. Thromb Haemost 2010; 104: 1258-1262.

9 Donze J, Le Gal G, Fine MJ, et al. Prospective validation of the Pulmonary Embolism Severity Index. A clinical prognostic model for pulmonary embolism. Thromb Haemost 2008; 100: 943-948.

10 Aujesky D, Roy PM, Le Manach CP. Validation of a model to predict adverse outcomes in patients with pulmonary embolism. Eur Heart J 2006; 27: 476-557.

11 Konstantinides SV, Torbicki A, Agnelli G, et al. 2014 ESC guidelines on the diagnosis and management of acute pulmonary embolism. Eur Heart J 2014; 35: 3033-3069.

12 Howard LSGE, Barden S, Condliffe R, et al. British Thoracic Society Guideline for the initial outpatient management of pulmonary embolism (PE). Thorax 2018; 73: Suppl. 2, ii1-ii29.

13 Meyer G, Vicaut E, Danays T, et al. Fibrinolysis for patients with intermediate-risk pulmonary embolism. $N$ Engl J Med 2014; 370: 1402-1411.

14 Society for Acute Medicine benchmarking audit (SAMBA17 report): against the clock-time for patients: a national audit of acute medical care in the UK. Edinburg, Society for Acute Medicine, 2017. www.acutemedicine.org.uk/ wp-content/uploads/2017/12/Society-for-Acute-Medicine-Benchmarking-Audit-2017-National-Report.pdf Date last accessed: March 19, 2019.

15 Douketis JD. Treatment of deep vein thrombosis: what factors determine appropriate treatment? Can Fam Physician 2005; 51: 217-223.

16 Kabrhel C, Okechukwu I, Hariharan P, et al. Factors associated with clinical deterioration shortly after PE. Thorax 2014; 69: 835-842.

17 Aujesky D, Stone RA, Kim S, et al. Length of hospital stay and postdischarge mortality in patients with pulmonary embolism: a statewide perspective. Arch Intern Med 2008; 168: 706-712.

18 Robertson L, Kesteven P, McCaslin JE. Oral direct thrombin inhibitors or oral factor Xa inhibitors for the treatment of pulmonary embolism. Cochrane Database Syst Rev 2015; 12: CD010957.

19 Ambulatory Emergency Care Network. Directory of Ambulatory Emergency Care for Adults. London, NHS Elect, 2018 www.ambulatoryemergencycare.org.uk/uploads/files/1/AEC-Directory\%206th\%20edition\%20February\% 202018.pdf Date last accessed: March 19, 2019.

20 Lim WS, van der Eerden MM, Laing R, et al. Defining community acquired pneumonia severity on presentation to hospital: an international derivation and validation study. Thorax 2003; 58: 377-382.

21 Pneumonia in adults: diagnosis and management (clinical guideline CG191). London, National Institute for Health and Care Excellence (NICE), 2014. www.nice.org.uk/guidance/cg191/resources/pneumonia-in-adultsdiagnosis-and-management-pdf-35109868127173 Date last updated: November, 2018. Date last accessed: March $19,2019$.

22 Jimenez D, Lobo JL, Monreal M, et al. Prognostic significance of multidetector CT in normotensive patients with pulmonary embolism: results of the protect study. Thorax 2014; 69: 109-115.

23 Furlan A, Aghayev A, Chang CC, et al. Short-term mortality in acute pulmonary embolism: clot burden and signs of right heart dysfunction at CT pulmonary angiography. Radiology 2012; 265: 283-293.

24 Jimenez D, Uresandi F, Otero R, et al. Troponin-based risk stratification of patients with acute nonmassive pulmonary embolism: systematic review and metaanalysis. Chest 2009; 136: 974-982.

25 Coutance G, Le Page O, Lo T, et al. Prognostic value of brain natriuretic peptide in acute pulmonary embolism. Crit Care 2008; 12: R109.

26 Moores L, Zamarro C, Gómez V, et al. Changes in PESI scores predict mortality in intermediate-risk patients with acute pulmonary embolism. Eur Respir J 2013; 41: 354-359.

27 Davies CW, Wimperis J, Green ES, et al. Early discharge of patients with pulmonary embolism: a two-phase observational study. Eur Respir J 2007; 30: 708-714.

28 Agterof MJ, Schutgens RE, Snijder RJ, et al. Out of hospital treatment of acute pulmonary embolism in patients with a low NT-proBNP level. J Thromb Haemost 2010; 8: 1235-1241. 